\title{
Restaurações cerâmicas delgadas sobre dentes sem preparo em diferentes regiões dos arcos dentais. Relato de 2 casos clínicos
}

Thin ceramic restorations on unprepared teeth in different regions of the dental arches. Report of 2 clinical cases Restauraciones cerámicas finas en dientes no preparados en diferentes regiones de los arcos dentales. Reporte de 2 casos clínicos

\author{
Danila de OLIVEIRA ${ }^{1}$ \\ Mariana Tassinari CAIXETA ${ }^{\mathbf{1}}$ \\ Fernando Isquierdo de SOUZA ${ }^{2}$ \\ Eduardo Passos ROCHA ${ }^{\mathbf{1}}$
}

${ }^{1}$ Departamento de Materiais Odontológicos e Prótese, Faculdade de Odontologia, UNESP, Universidade Estadual Paulista, 16015-050 Araçatuba - SP, Brasil ${ }^{2}$ Curso de Odontologia, Campus Jacarezinho, UENP, Universidade Estadual do Norte do Paraná 86400-000 Jacarezinho - PR, Brasil

\begin{abstract}
Resumo
As reforçadas propriedades intrínsecas dos materiais cerâmicos, associados à adesão resinosa fundamentam a desenvoltura de técnicas restauradoras mais simplificadas, conservadoras e sem a necessidade de preparo dental prévio seja em dentição anterior ou posterior. Assim sendo, o trabalho tem como objetivo apresentar dois casos clínicos os quais abordaram a confecção de restaurações delgadas em dissilicato de lítio cimentadas sobre diferentes áreas dos arcos dentários, enfatizando o mesmo protocolo clínico, ou seja, ausência de preparos dentais e consequentemente a cimentação resinosa adesiva das restaurações. Caso Clínico 1: laminados cerâmicos nos elementos 13, 12, 11, 21, 22 e 23; Caso Clínico 2: facetas delgadas sobre os elementos 34, 35, 36 e 37. Ambos os casos clínicos com 1 ano de acompanhamento após a cimentação das restaurações. Conclui-se que houve a recuperação da estética e da função em ambos os casos apresentados e a confiabilidade e o sucesso em longo prazo do protocolo clínico descrito estão diretamente relacionados ao substrato dentário, aos fatores de adesividade dos materiais e as propriedades intrínsecas das cerâmicas.
\end{abstract}

Descritores: Cerâmica; Estética Dentária; Facetas Dentárias; Cimentos de Resina.

\section{Abstract}

The reinforced intrinsic properties of the ceramic materials, associated with the resinous adhesion, support the development of restorative techniques that are simplified, conservative and without the need of previous dental preparation, either in anterior or posterior dentition. The objective of this study was to present two clinical cases which used thin restorations in lithium disilicate cemented in different areas of the dental arches, emphasizing the same clinical protocol, that is, the absence of dental preparations and, consequently, resinous cementation. Clinical Case 1: ceramic laminates veneers in elements 13, 12, 11, 21, 22 and 23; Clinical case 2: thin ceramic restorations on elements 34, 35,36 and 37. At both cases with 1 year of follow-up after cementation of the restorations. It was concluded that there was recovery of aesthetics and function in both cases presented and the reliability and long-term success of the described clinical protocol are directly related to the dental substrate, the material adhesion factors and the intrinsic properties of the ceramics.

Descriptors: Ceramics; Esthetics, Dental; Dental Veneers; Resin Cements.

\section{Resumen}

Las propiedades intrínsecas reforzadas de los materiales cerámicos, asociadas con la adhesión resinosa, apoyan el desarrollo de técnicas restaurativas que son simplificadas, conservadoras y sin la necesidad de una preparación dental previa, ya sea en la dentición anterior o posterior. El objetivo de este estudio fue presentar dos casos clínicos que utilizaron restauraciones delgadas en disilicato de litio cementadas en diferentes áreas de los arcos dentales, enfatizando el mismo protocolo clínico, es decir, la ausencia de preparaciones dentales y, en consecuencia, la cementación resinosa. Caso Clínico 1: carillas cerámicas en los elementos 13, 12, 11, 21, 22 y 23; Caso clínico 2: restauraciones cerámicas delgadas en los elementos 34, 35, 36 y 37. Ambos casos con 1 año de seguimiento después de la cementación de las restauraciones. Se concluyó que hubo recuperación de la estética y la función en ambos casos presentados y la fiabilidad y el éxito a largo plazo del protocolo clínico descrito están directamente relacionados con el sustrato dental, los factores de adhesión del material y las propiedades intrínsecas de la cerámica.

Descriptores: Cerámica; Estética Dental; Coronas con Frente Estético; Cementos de Resina;

\section{INTRODUÇÃO}

A maior exigência e a crescente busca dos pacientes por tratamentos dentários estéticosfuncionais somado ao aperfeiçoamento dos materiais dentários tem contribuído para a difusão da Odontologia minimamente invasiva, a qual tem como objetivo principal preservar, ao máximo, a estrutura dental sadia ${ }^{1-3}$. Devido a isso, a Odontologia atual permite a reabilitação do paciente com a utilização de restaurações indiretas cimentadas sobre dentes com mínimo ou nenhum desgaste da estrutura dentária ${ }^{4-6}$.

Dentro desse contexto, as cerâmicas possuem destaque cada vez maior na odontologia restauradora devido as suas excelentes características ópticas, biomiméticas, biocompatibilidade, longevidade e resistência mecânica mesmo em restaurações com espessuras muito delgadas ${ }^{3,7-9}$. E, dentre os diferentes sistemas cerâmicos, o dissilicato de lítio pode ser hábil para satisfazer as diferentes propostas restauradoras e estéticas, em dentição anterior ou posterior, sejam elas coroas, facetas, laminados ou fragmentos cerâmicos ${ }^{6,7,10,11}$. No mais, a utilização de sistemas adesivos e cimentação resinosa aliados a preservação de esmalte dentário como substrato, elevaram significativamente as taxas de sucesso mecânico deste tipo de restauração ${ }^{7,12,13}$.

Com isto, o objetivo do presente trabalho é relatar dois casos clínicos que reúnem características desafiadoras para a obtenção do resultado final no que concerne à restauração dos elementos com restaurações cerâmicas delgadas em dissilicato de lítio, sem a necessidade de preparos dentais prévios, abordando a técnica minimamente invasiva em diferentes situações clinicas e fundamentadas dentro de um único protocolo de atuação.

\section{CASO CLÍNICO 1}

Paciente do sexo masculino, 20 anos de idade, queixava-se do formato dos dentes anteriores da maxila. Além disso, relatava insegurança ao executar suas atividades cotidianas como, por exemplo, conversar e sorrir. 
No exame clínico foi notado que, de forma geral, a estética do sorriso era insatisfatória por conta da presença de diastemas em toda a dentição maxilar anterior (Figura 1), sendo assim, determinou-se como plano de tratamento a confecção de laminados cerâmicos nos elementos $13,12,11$,21, 22 e 23, embasando-se nos aspectos clínicos associados aos dados do enceramento diagnóstico.

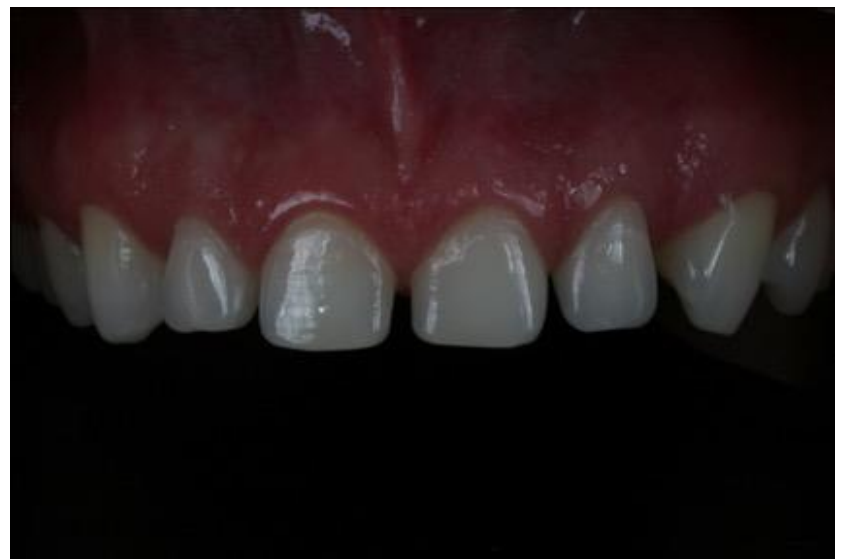

Figura 1: Aspecto inicial do caso, evidenciando a presença de diastemas nos dentes anteriores superiores.

\section{CASO CLÍNICO 2}

Paciente do sexo feminino, 40 anos, queixava-se de desconforto nos dentes posteriores, oriundos de prováveis fraturas que a paciente mencionou ter ocorrido ao longo do tempo.

Durante o exame clínico observou-se a presença de desgastes severos nas faces oclusais dos elementos 34, 35, 36 e 37 com fraturas oclusais que causavam comprometimento anatômico e fisiológico dos dentes envolvidos. Entretanto, os elementos apresentavam bom remanescente dental (Figura 2).

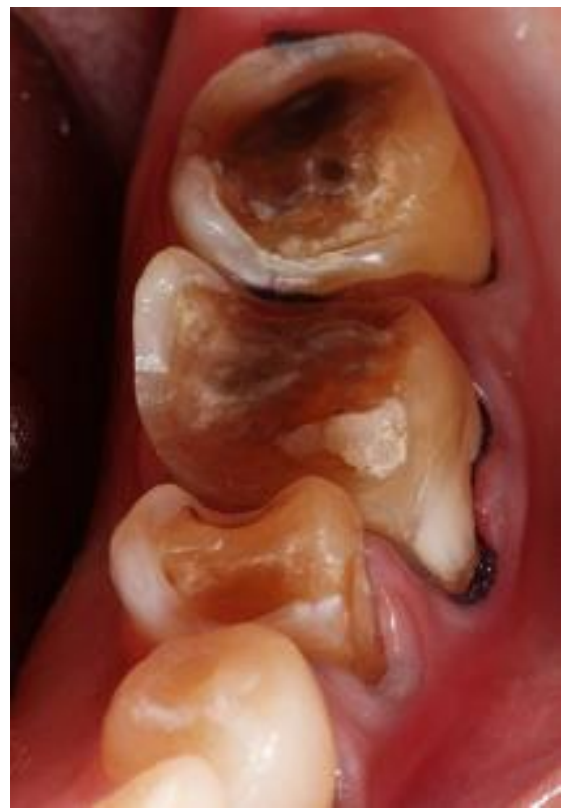

Figura 2: Aspecto inicial do caso, evidenciando a presença de desgastes e fraturas nos dentes 34 a 37.

Como o remanescente dental expressava adequada expulsividade e ideal eixo de inserção, foi determinado como plano de tratamento, a realização de restaurações em dissilicato de lítio, sem nenhum tipo de redução dental, nos elementos 34, 35, 36 e 37.

\section{PROTOCOLO CLÍNICO}

Muito embora houvesse uma diferença entre a variação dos elementos envolvidos em cada caso, o tipo de abordagem foi o mesmo para as duas situações: ausência de desgastes dentais, uma vez que se preconizou a racionalização de estruturas, e o uso de restaurações em dissilicato de lítio (e-Max, IPS e.Max Press; Ivoclar Vivadent, Schaan, Liechtenstein) com a realização de um mesmo protocolo de moldagem e cimentação, independente da localização do dente no arco dentário.

As moldagens de diagnóstico foram realizadas com silicone por adição (Express XT, 3M ESPE, St. Paul, MN, USA) com o uso de material denso e leve. Os modelos de estudo foram montados em articulador semi-ajustável (Bio-Art, São Carlos, SP, Brasil) e encaminhados ao laboratório de prótese dentária para a obtenção do enceramento diagnóstico.

Excepcionalmente para o caso clínico 1, foi realizado o ensaio estético, "mock-up", com o objetivo de confirmar o plano de tratamento ${ }^{14}$. Adicionalmente foram confeccionadas duas máscaras de silicone por condensação (Zetaplus/OranWash, Zhermack, Roma, Italia) através da cópia do enceramento diagnóstico, sendo uma no plano vertical e outra no plano horizontal, para verificar o espaço disponível para a restauração ${ }^{15}$.

$\mathrm{Na}$ sequência do tratamento, os elementos foram polidos com auxílio de discos de lixa para acabamento de restaurações em resina (Sof-lex Popon, 3M ESPE, St. Paul, MN, USA) apenas com a finalidade de arredondar possíveis ângulos vivos e melhorar o aspecto da superfície do substrato. As moldagens de trabalho foram realizadas com silicone por adição (Express XT, 3M ESPE, St. Paul, MN, USA) através da técnica de moldagem simultânea com dois fios afastadores não impregnados (Ultrapack, Ultradent Products, South Jordan, Utah, USA) de calibre compatível com o biótipo gengival de cada paciente ${ }^{16}$. Em seguida, foi realizada a orientação do arco facial para a montagem do modelo de trabalho maxilar no articulador semiajustável, o registro maxilo-mandibular por justaposição manual $\mathrm{e}$, em conjunto com as fotografias digitais, foram encaminhados ao laboratório de prótese dentária. Para o processo de seleção de cor foi utilizada a escala Vita Classical (VITA Zahnfabrik GmbH, Bad Säckingen, Germany) na sequência de luminosidade.

Ao receber as restaurações do laboratório de prótese, as quais apresentavam espessuras que variavam de 0,3mm até $0,7 \mathrm{~mm}$ (Caso Clínico 1 e 2), foi realizada a "prova seca" das restaurações para visualizar a adaptação de cada peça ao dente com o auxílio de sonda exploradora (Hu-Friedy Manufacturing, Chicago, IL, USA). Depois dessa 
fase, realizou a "prova úmida" com o uso das pastas de prova (Rely X Veneer Try-in, 3M ESPE, Seefeld, Germany), para a verificação da luminosidade e do melhor matiz do cimento a ser utilizado na etapa de cimentação. O preparo das peças cerâmicas obedeceu a sequência: condicionamento ácido da superfície interna das restaurações com ácido fluorídrico à $10 \%$ durante 20 segundos, lavagem com água e secagem, aplicação do ácido fosfórico à $37 \%$ por 1 minuto, lavagem com água e secagem e aplicação de silano (Rely X Ceramic Primer, 3M ESPE, St. Paul, MN, USA) ${ }^{16}$, aguardando a evaporação do solvente, conforme recomendação do fabricante. Os dentes foram condicionados com ácido fosfórico à 37\% durante 20 segundos, lavagem com água e secagem e aplicação de uma camada de adesivo (Single\&Bond 2.0) e remoção dos excessos com jato de ar por 10 segundos, sob isolamento relativo ${ }^{17}$.

O cimento Rely X Veneer (Rely X Veener, 3M ESPE, Seefeld, Germany) foi aplicado diretamente sobre a superfície interna das restaurações e as mesmas foram corretamente posicionadas sobre os dentes. Após exposição de luz para iniciar a fotopolimerização, por 5 segundos em cada face (vestibular, palatina, interproximais e oclusal) os excessos de cimento foram removidos com auxílio de uma sonda exploradora e fio dental nas faces proximais. Depois disso, cada face recebeu luz por mais 40 segundos para completa fotopolimerização do cimento (VALO, Ultradent Products Inc, South Jordan, UT, USA). O aspecto final das restaurações após 1 ano de acompanhamento clínico pode ser visualizado nas Figuras 3 e 4.

\section{DISCUSSÃO}

A Odontologia restauradora, os materiais dentários e o perfil dos pacientes que buscam tratamentos restauradores têm mudado consideravelmente nos últimos anos, sendo que o restabelecimento da função, assim como da estética devem ser levados em consideração em todos os $\operatorname{casos}^{18-20}$.

Um grande número de autores sugere que a manutenção de estrutura dental nos preparos para restaurações cerâmicas eleva o sucesso dos tratamentos ${ }^{21-24}$, de modo que a associação entre cimentos resinosos e os atuais procedimentos de preparos do dente e da peça cerâmica, aumentaram em aproximadamente $69 \%$ a resistência à fratura da restauração em comparação ao uso de cimentos convencionais não resinosos ${ }^{25}$. Ainda, estudos clínicos com acompanhamento ao longo de 10 anos relatam falhas adesivas entre a superfície dental e o cimento resinoso, possivelmente atribuídas à extensão do preparo dental, particularmente nos preparos profundos em dentina ${ }^{26-29}$. Segundo os mesmos autores, falhas entre o cimento e o esmalte raramente foram observadas.

Além disso, a resistência de união também é afetada pelo sistema adesivo ${ }^{26}$. Sistemas adesivos que utilizam a técnica de condicionamento de três passos detém melhores resultados de resistência de união em comparação com os adesivos autocondicionantes ${ }^{26,30}$, ressaltando que o substrato em esmalte é sabidamente menos sensível ao condicionamento com ácido fosfórico a $37 \%$ que a dentina no que diz respeito à hipersensibilidade dental e tempo excessivo de condicionamento ${ }^{23}$.

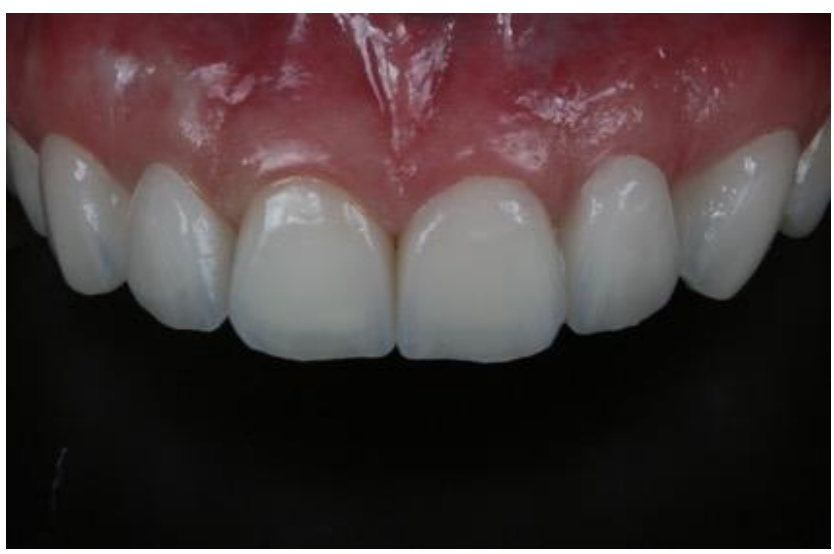

Figura 3: Acompanhamento de 1 ano após a cimentação dos laminados cerâmicos.

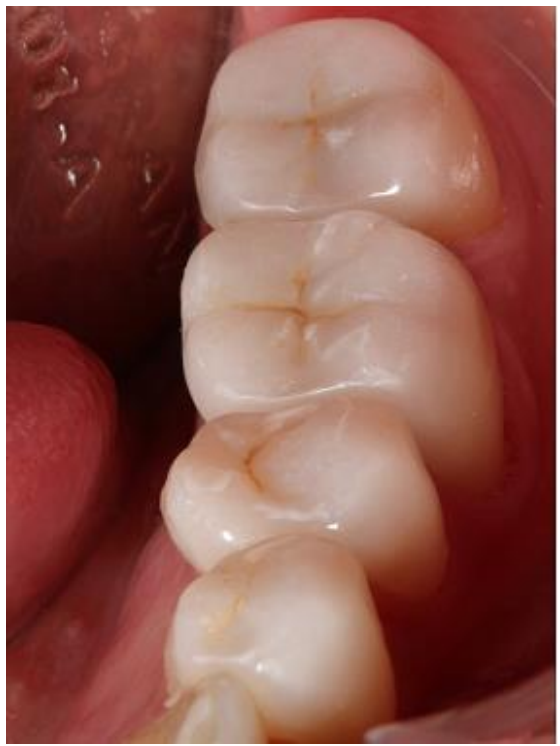

Figura 4: Acompanhamento de 1 ano após a cimentação das restaurações cerâmicas.

Com relação ao sistema cerâmico, o dissilicato de lítio tem sido amplamente utilizado em razão das suas excelentes características estéticas, com o índice de refração de luz próximo ao do esmalte dentário associado a uma alta resistência mecânica, com indicação nos mais diversos casos de prótese dentária sendo possível, nos dias atuais, o uso do dissilicato de lítio com os protocolos clínicos que minimizam ou dispensam os preparos dentais ${ }^{7-11}$, como em ambos os casos apresentados.

Dessa maneira, foi realizada uma abordagem menos invasiva na qual se preconizou a preservação do esmalte como substrato, a fim de conferir maior 
resistência de união à interface dente - cimento resinoso - restauração cerâmica, minimizando possíveis intercorrências pós-operatórias, o que possibilita ao tratamento minimamente invasivo maior previsibilidade de sucesso clínico.

\section{CONCLUSÃO}

Houve a recuperação da estética e da função em ambos os casos apresentados e a confiabilidade e o sucesso em longo prazo do protocolo clínico descrito estão diretamente relacionados ao substrato dentário, aos fatores de adesividade dos materiais e as propriedades intrínsecas das cerâmicas.

\section{REFERÊNCIAS}

1. Murdoch-Kinch CA, McLean ME. Minimally invasive dentistry. J Am Dent Assoc. 2003;134(1):87-95.

2. Ericson D. The concept of minimally invasive dentistry. Dent Update. 2007;34(1):9-10, 12-4, 17-8.

3. Beier US, Kapferer I, Burtscher D, Dumfahrt H. Clinical performance of porcelain laminate veneers for up to 20 years. Int $\mathrm{J}$ Prosthodont. 2012;25(1):79-85.

4. D'Arcangelo C, Vadini M, D'Amario M, Chiavaroli Z, De Angelis F. Protocol for a new concept of no-prep ultrathin ceramic veneers. J Esthet Restor Dent. 2018;30(3):173-79.

5. Vadini M, D'Amario M, De Angelis F, Falco A, D'Arcangelo C. No-prep rehabilitation of fractured maxillary incisors with partial veneers. $\mathrm{J}$ Esthet Restor Dent. 2016;28(6):351-58.

6. Molina IC, Molina GC, Stanley K, Lago C, Xavier CF, Volpato CA. Partial-prep bonded restorations in the anterior dentition: long-term gingival health and predictability. A case report. Quintessence Int. 2016;47(1):9-16.

7. Kelly JR, Benetti P. Ceramic materials in dentistry: historical evolution and current practice. Aust Dent J. 2011;56(Suppl 1):84-96.

8. Edelhoff D, Brix O. All-ceramic restorations in different indications: a case series. J Am Dent Assoc. 2011;142(Suppl 2):14S-9S.

9. Miranda ME, Olivieri KA, Rigolin FJ, Basting RT. Ceramic fragments and metal-free full crowns: a conservative esthetic option for closing diastemas and rehabilitating smiles. Oper Dent. 2013;38(6):567-71.

10. Cortellini D, Canale A. Bonding lithium disilicate ceramic to feather-edge tooth preparations: a minimally invasive treatment concept. J Adhes Dent. 2012;14(1):7-10.

11. Wolfart S, Eschbach S, Scherrer S, Kern M. Clinical outcome of three-unit lithium-disilicate glass-ceramic fixed dental prostheses: up to 8 years results. Dent Mater. 2009;25(9):e63-71.

12.Benetti P, Della Bona A, Kelly JR. Evaluation of thermal compatibility between core and veneer dental ceramics using shear bond strength test and contact angle measurement. Dent Mater. 2010;26(8):743-50.

13.Beier US, Kapferer I, Dumfahrt H. Clinical longterm evaluation and failure characteristics of 1,335 all-ceramic restorations. Int J Prosthodont. 2012;25(1):70-8.

14. Veneziani M. Ceramic laminate veneers: clinical procedures with a multidisciplinary approach. Int J Esthet Dent. 2017;12(4):426-48.

15. Anchieta RB, Rocha EP, de Almeida EO, Junior AC, Martini AP. Bonding all-ceramic restorations with two resins cement techniques: a clinical report of three-year follow-up. Eur J Dent. 2011;5(4):478-85.

16.Peumans M, Van Meerbeek B, Lambrechts P, Vanherle G. Porcelain veneers: a review of the literature. J Dent. 2000;28(3):163-77.

17.Barkmeier WW, Erickson RL, Kimmes NS, Latta MA, Wilwerding TM. Effect of enamel etching time on roughness and bond strength. Oper Dent. 2009;34(2):217-22.

18.Esquivel-Upshaw J, Rose W, Oliveira E, Yang M, Clark AE, Anusavice K. Randomized, controlled clinical trial of bilayer ceramic and metal-ceramic crown performance. J Prosthodont. 2013; 22(3):166-73.

19. Martin AJ, Buschang PH, Boley JC, Taylor RW, McKinney TW. The impact of buccal corridors on smile attractiveness. Eur J Orthod. 2007;29(5):530-7.

20.Fradeani M. Evaluation of dentolabial parameters as part of a comprehensive esthetic analysis. Eur J Esthet Dent. 2006;1(1):62-9.

21.Borges GA, Sophr AM, de Goes MF, Sobrinho LC, Chan DC. Effect of etching and airborne particle abrasion on the microstructure of different dental ceramics. J Prosthet Dent. 2003; 89(5):479-88.

22.Fabianelli A, Goracci C, Bertelli E, Davidson CL, Ferrari M. A clinical trial of Empress II porcelain inlays luted to vital teeth with a dual-curing adhesive system and a self-curing resin cement. J Adhes Dent. 2006;8(6):427-31.

23. Aykor A, Ozel E. Five-year clinical evaluation of 300 teeth restored with porcelain laminate veneers using total-etch and a modified self-etch adhesive system. Oper Dent. 2009;34(5):516-23.

24.Oztürk E, Hickel R, Bolay S, Ilie $\mathrm{N}$. Micromechanical properties of veneer luting resins after curing through ceramics. Clin Oral Investig. 2012;16(1):139-46.

25.Scherrer SS, de Rijk WG, Belser UC, Meyer JM. Effect of cement film thickness on the fracture resistance of a machinable glass-ceramic. Dent Mater. 1994;10(3):172-77.

26. Gresnigt M, Ozcan M. Esthetic rehabilitation of anterior teeth with porcelain laminates and 
sectional veneers. J Can Dent Assoc. 2011;77:b143

27.Friedman MJ. A 15-year review of porcelain veneer failure--a clinician's observations. Compend Contin Educ Dent. 1998;19(6):625-8, 630, 632 passim;quiz 638.

28.Peumans M, De Munck J, Fieuws S, Lambrechts P, Vanherle G, Van Meerbeek B. A prospective ten-year clinical trial of porcelain veneers. J Adhes Dent. 2004;6(1):65-76.

29.Fradeani M, Redemagni M, Corrado M. Porcelain laminate veneers: 6- to 12-year clinical evaluation--a retrospective study. Int $\mathrm{J}$ Periodontics Restorative Dent. 2005;25(1):9-17.

30.De Munck J, Van Landuyt K, Peumans M, Poitevin A, Lambrechts P, Braem $\mathrm{M}$ et al. A critical review of the durability of adhesion to tooth tissue:methods and results. J Dent Res. 2005;84(2):118-32.
CONFLITO DE INTERESSES

Os autores declaram não haver conflitos de interesse.

\section{AUTOR PARA CORRESPONDENCIA}

\section{Eduardo Passos Rocha}

1rocha.eduardo@gmail.com

Submetido em 09/06/2018

Aceito em 04/10/2018 